\title{
PRECISANDO LA CRONOLOGÍA PARA LA INHUMACIÓN TIPO CHENQUE, VALLE DEL RÍO CISNES (AISÉN, CHILE), PATAGONIA CENTRAL
}

\author{
OMAR REYES* Y CESAR MÉNDEZ*
}

\begin{abstract}
RESUMEN
Las investigaciones realizadas a la fecha en el valle del río Cisnes en la Región de Aisén carecían de información bioantropológica. El reciente hallazgo de un chenque disturbado con osamentas humanas entrega datos en torno a estos contextos fúnebres, incluyendo información cronológica e isotópica. La mayoría de las edades conocidas para este tipo de entierros a cielo abierto en Pampa-Patagonia se circunscriben al último milenio. Los resultados obtenidos en Pampa El Frío se suman a otros que permiten retrotraer en cerca de 400 años este patrón mortuorio.
\end{abstract}

PALABRAS CLAVES: restos humanos, chenques, cronología, Patagonia central.

\section{PRECISING THE CHRONOLOGY FOR THE CHENQUE BURIAL-TYPE, CISNES RIVER BASIN (AISÉN, CHILE), CENTRAL PATAGONIA}

\begin{abstract}
Current research at Cisnes river basin lacked bioanthropological information. The recent finding of a disturbed chenque bearing human remains provides data related to these funerary contexts, including chronological and isotopic information. The majority of known ages for this open-air burial-type in Pampa-Patagonia are limited to the last millennium. Results obtained at Pampa El Frío and others take back this mortuary pattern in 400 years.
\end{abstract}

KEYWORDS: human remains, chenques, chronology, central Patagonia.

Investigador adjunto Centro de Estudios del Hombre Austral, Instituto de la Patagonia, Universidad de Magallanes. Av. Bulnes 1890, Punta Arenas, Chile. omarreyesbaez@gmail.com

* Departamento de Antropología, Facultad Ciencias Sociales, Universidad de Chile. Ignacio Carrera Pinto 1045, Nuñoa, Santiago, Chile. cmendezm@uchile.cl 


\section{INTRODUCCIÓN}

El patrón inhumatorio tipo chenque, ha sido ampliamente documentado en Pampa-Patagonia, tanto histórica como arqueológicamente, (pe. Berón et al. 2000, De La Vaulx 1901, Goñi y Barrientos 2000, Prieto 1993-94, Reyes 2001, 2002), como una de las modalidades de entierros más comunes practicadas por los grupos de cazadores recolectores durante el Holoceno Tardío, especialmente a partir del último milenio (Castro y Moreno 2000, Goñi 2001, Berón y Baffi 2004). En la Región de Aisén, en el margen occidental de Patagonia central, el registro de este tipo de contextos ha sido más bien discreto (Goñi et al. 2004, Mena y Lucero 2004, Reyes 2001, 2002). Por una parte, las prospecciones sistemáticas llevadas a cabo en algunos de sus principales valles (Chacabuco, Jeinemeni, Ibáñez) avalan una notoria menor densidad, y por otra, las escasas evidencias documentadas se encuentran en su mayoría disturbadas (Reyes 2004). En el valle del río Cisnes en tanto, las prospecciones y sondeos permitieron el registro de una serie de acumulaciones intencionales de piedra similares a los chenques ${ }^{1}$ en baja frecuencia (Fig. 1). Sin embargo, la diferencia radica principalmente en que todos los rasgos tipo chenque se encontraron disturbados, sin ningún tipo de evidencia bioantropológica (Reyes et al. 2006).

El reciente hallazgo de un chenque disturbado conteniendo osamentas humanas en Pampa El Frío, en el curso superior del valle del río Cisnes, nos

1 "Esto ha sido explicado alternativamente como marcadores territoriales (Goñi y Barrientos 2000, Reyes 2004), hitos de colonos o como chenques "falsos" para evitar el saqueo indiscriminado de las tumbas por parte de colonos. Esto último, porque una actitud parecida es mencionada por De La Vaulx (1901), quién describe a grupos indígenas ocultando intencionalmente un cadáver (no conformando un chenque) para que éste no sea visto y profanado. Bien podría tratarse de actitudes con consecuencias materiales distintas para cumplir un mismo fin. No obstante, es necesario advertir que también es esperable una pobre conservación..., lo que se traduce en la desaparición frecuente de los restos orgánicos. En síntesis, todas las acumulaciones registradas... son intencionales, pero su función (potencialmente ritual) es sólo discriminable a partir de su intervención arqueológica. No existe otra manera, ni por asociación con otros sitios, ni por disposición en el paisaje, de resolver alternativamente esta asignación." (Reyes et al. 2006:82).

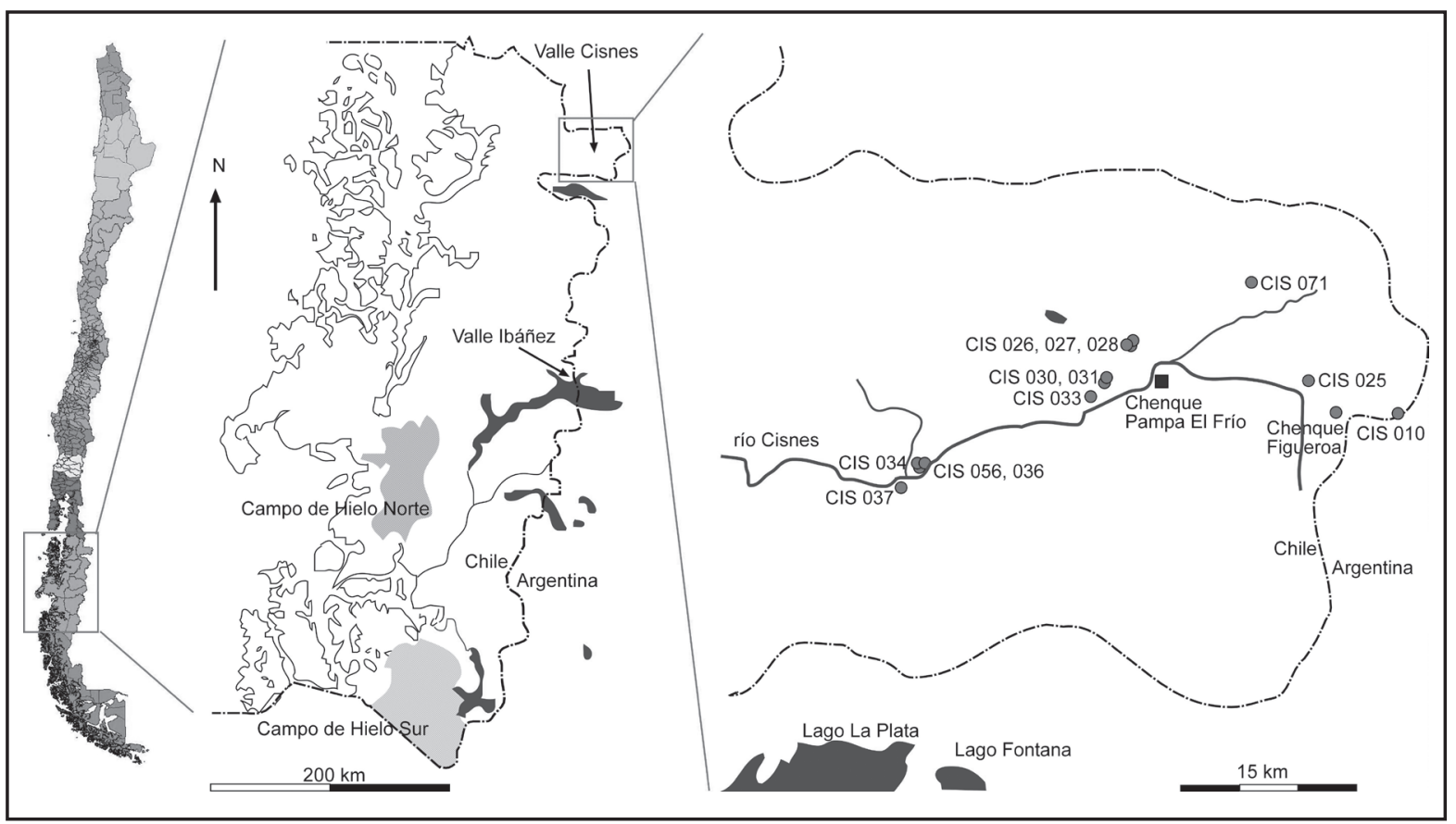

Fig. 1. Mapa de la Región de Aisén (izquierda) y el valle del río Cisnes (derecha), mostrando el sitio de hallazgo (Pampa El Frío: cuadrado) y otras localidades discutidas en el texto con acumulaciones intencionales de piedras (círculos). 
permitió realizar un relevamiento de información del que hasta ahora carecíamos ${ }^{2}$. Este reporte incorpora el estudio bioantropológico de los restos esqueletales recuperados, además de edades ${ }^{14} \mathrm{C}$ directas y datos de los isotopos ${ }^{13} \mathrm{C} y{ }^{15} \mathrm{~N}$. Estos análisis se enmarcan en los objetivos de un proyecto de investigación en curso en tanto proveen datos bioantropológicos e isotópicos comparativos, permiten precisar la cronología de la ocupación humana del valle y ayudan a caracterizar las distintas etapas del proceso de poblamiento de la región.

\section{MATERIAL Y METODOLOGÍA}

Se registraron restos óseos de la porción craneal (restaurada en el Servicio Médico Legal de Santiago) y postcraneal. En cuanto al cráneo, falta la porción izquierda de los huesos zigomático, nasal y palatino además de parte del parietal del mismo lado. Casi todas las piezas dentales del maxilar se han perdido postmortem con excepción del primer molar derecho y el segundo molar del mismo lado (perdido premortem). La mandíbula en tanto, se encuentra erosionada y fragmentada en dos partes. Se han perdido ambos cóndilos mandibulares y todas las piezas dentales con excepción de los terceros molares. Respecto al postcráneo, se recuperaron tres fragmentos de diáfisis de húmero, las diáfisis de ambos fémures y un fragmento de diáfisis de la tibia derecha, además de una serie de capas corticales de hueso de procedencia indeterminada.

Para realizar el análisis bioantropológico, se efectuaron una serie de observaciones macroscópicas (Buikstra y Ubelaker 1994) sobre la morfología de las diferentes porciones de la estructura ósea que fueron recuperadas, con el fin de determinar el sexo y edad de muerte (Brooks y Suchey 1990, Meindl y Lovejoy 1985), registrar rasgos discretos (Gill y Rhine 1990) y paleopatologías observables (Ortner

2 Debido a una denuncia efectuada a la Fiscalía Regional por el hallazgo de osamentas humanas, la Policía de Investigaciones de Chile realizó el levantamiento de los restos, remitiéndolos posteriormente al Servicio Médico Legal (2009). Dicho levantamiento, omitió un registro básico sobre disposición de los restos, contexto y rasgos notables. No obstante, es posible sugerir que se trata de una modalidad de entierro tipo chenque ya que su descubridor, el Sr. Andrés Hernández, ha sido ayudante en los trabajos arqueológicos efectuados por los diferentes programas de investigación en el valle, conociendo ampliamente este tipo de contextos.
2003). Finalmente, cabe señalar, que un diente se utilizó para fechación ${ }^{14} \mathrm{C}$ AMS y análisis de trazas isotópicas ${ }^{13} \mathrm{C}$ y ${ }^{15} \mathrm{~N}$ (Ambrose y Katzenberg 2000).

\section{RESULTADOS}

Dado lo escaso y fragmentado de los restos óseos no fue posible utilizar la mayoría de los métodos existentes para estimar la edad y sexo del individuo. Sin embargo, la observación de la morfología craneal, obliteración de las suturas ectocraneales, junto al desarrollo y desgaste de la dentición, permiten sugerir que los restos corresponden a una persona adulta, de sexo masculino, cuya rango de edad al morir se estima alrededor de los $35 \pm 5$ años, con rasgos discretos craneales y postcraneales asignables a la población amerindia.

Por otro lado, la observación macroscópica de los huesos del cráneo y postcráneo permite inferir modos de vida relacionados con prácticas cazadorasrecolectoras. Es así como las piezas dentales presentan las consecuencias de una dieta abrasiva, un estrés mecánico prolongado y el comienzo de patologías periodontales. En efecto, las patologías orales detectadas corresponden a la pérdida del segundo molar derecho del maxilar producto de un gran absceso. El primer molar del mismo lado presenta desgaste dental con eliminación de cúspides y esmalte, además de una gran caries en norma oclusal. La pieza presenta retracción alveolar severa, con más de dos tercios de la raíz expuesta, y evidencias que sugieren que también fue afectada por el absceso mencionado. El paladar presenta actividad porótica probablemente relacionada a este proceso infeccioso $e$ inflamatorio. Los terceros molares mandibulares también presentan desgaste dental con eliminación del esmalte y cúspides coronales, además de exposición de dentina secundaria. La retracción alveolar es ligera, exponiendo menos de la mitad de la raíz de las piezas dentales. No se observó tártaro dental en estas piezas. Adicionalmente, el gran desarrollo de la tuberosidad zigomaxilar sugiere que la arcada dental fue utilizada como apoyo de algunas actividades (p.e. curtir, raer); lo que sumado al desgaste dental, avala el intenso uso y estrés al que fue sometida la cavidad bucal.

Finalmente, se registró un trauma en el torus supraorbital. Se trata de una pequeña hendidura en forma semiovalada de 8 por $4 \mathrm{~mm}$. Ésta, obedece a 
un golpe que afecto la tabla externa del hueso (periosteo). El trauma, de origen desconocido, regeneró meses antes de la muerte del individuo, notándose sólo una leve actividad porótica alrededor del golpe.

Un tercer molar izquierdo otorgó una edad ${ }^{14} \mathrm{C}$ de $1.590 \pm 25$ años AP. (1.534 a 1411 años cal AP. a un $95,4 \%$ y valores de $\delta^{13} \mathrm{Ccol}$ de $-19.6 \%$ y $\delta^{15} \mathrm{Ncol}$ de $10.7 \%$ (UGAMS 5963).

\section{DISCUSIÓN Y CONCLUSIONES}

De acuerdo al relevamiento realizado del contexto inhumatorio hallado en Pampa El Frío es posible establecer que correspondería a una inhumación del tipo chenque a cielo abierto o acumulación intencional de piedras a manera de túmulo sobre el cuerpo del individuo. No es posible aseverar si el cuerpo se encontraba bajo la superficie o sobre ella, previo a ser tapado. La orientación, disposición del cuerpo y la existencia de otros rasgos (p.e. fogones, ocre) y ofrendas es desconocida.

Los restos humanos recuperados, corresponden a un solo individuo, de sexo masculino, con una edad estimada al morir de $35 \pm 5$ años. Se observó un pequeño trauma craneal y evidencias de patologías orales producto de múltiples factores (falta de limpieza, inflamación e infección del hueso cortical) que sugieren una dieta abrasiva propia de grupos cazadores recolectores terrestres y el uso de la boca como ayuda en algunas actividades. Los resultados isotópicos, al ser comparadas con los niveles y promedios de otras poblaciones arqueológicas y la ecología isotópica de algunos recursos de Pampa y Patagonia (Barberena 2002, Berón et al. 2009, Tessone et al. 2005, 2009), respaldan una dieta continental terrestre, vinculada a la vía fotosintética $\mathrm{C}_{3}$.

Por otro lado, la edad ${ }^{14} \mathrm{C}$ de 1.590 años AP. obtenida de las osamentas de Pampa El Frío ayuda a precisar la antigüedad del patrón tipo chenque. Ésta se suma a las fechas de ca.1.030 años AP. para este tipo de entierros en La Pampa (Berón y Baffi 2004), ca. 1.140 años AP. en Patagonia centromeridional (Goñi 2001, Goñi et al. 2004, Goñi y Barrientos 2004, Cassiodoro y García 2009) y ca. 1.155 años AP. para Magallanes (Morano et al. 2009). Esta distancia cronológica, se acentúa aún más al compararla con los únicos fechados de restos

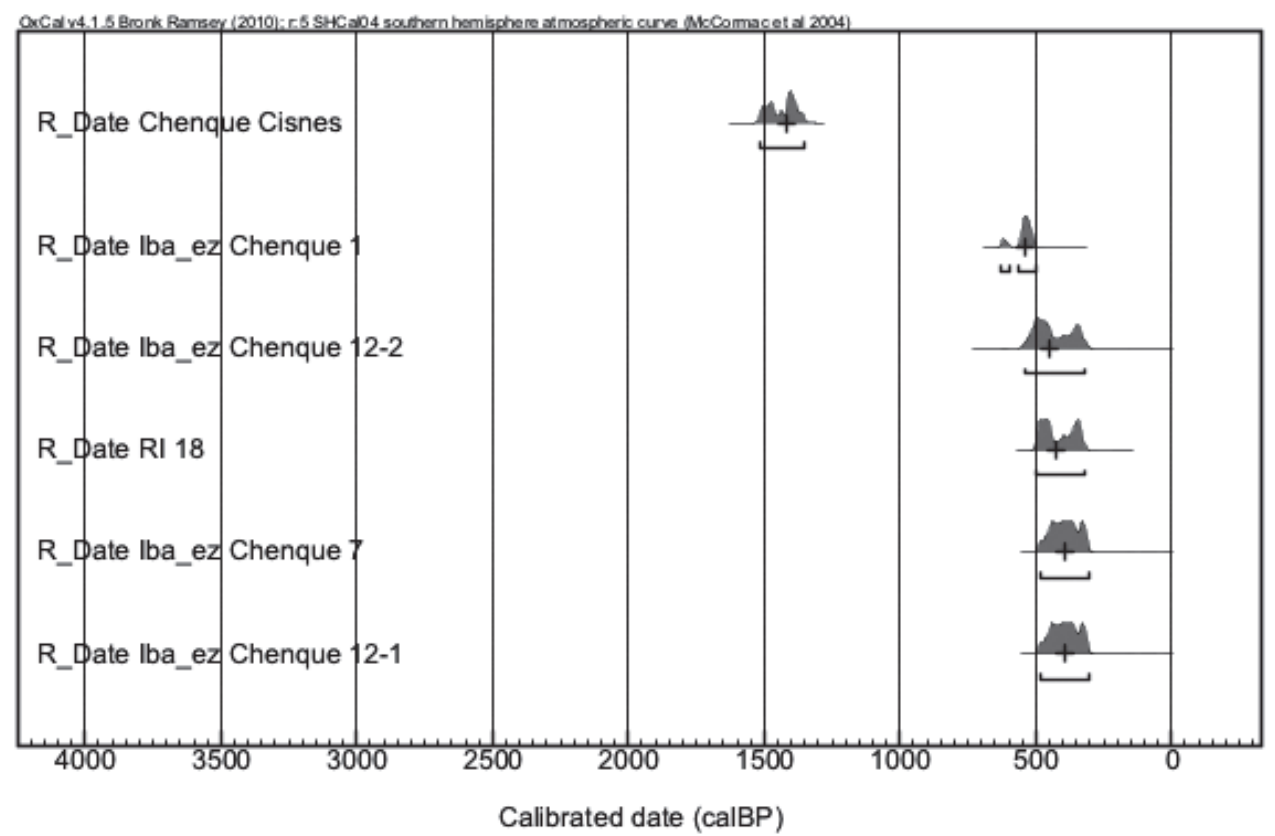

Fig. 2. Síntesis de edades ${ }^{14} \mathrm{C}$ calibradas sobre restos humanos tardíos de Aisén. La primera edad (arriba) corresponde a las evidencias de Pampa El Frío en el curso superior del valle del río Cisnes, mientras que todas las demás, se ubican en el curso inferior del valle del río Ibáñez. Todos los contextos son chenques, excepto RI18 que corresponde a un entierro bajo reparo rocoso (Reyes 2001). Las edades fueron calibradas con un rango de $2 \delta$ con el programa OxCal 4.1 con la información de la curva ShCal 04 (Bronk Ramsey 2009). 
humanos procedentes de chenques de la Región de Aisén (figura 1), ya que 200 km más al Sur, en la desembocadura del río Ibáñez, se ha podido fechar consistentemente este patrón entre los 650 y 300 años cal. AP. (Reyes 2001, 2002. Figura 2).

\section{AGRADECIMIENTOS}

Financiamiento FONDECYT 1090027. Agradecemos la ayuda del Sr. Andrés Hernández, a la administración y personal de la Estancia Río Cisnes, al Servicio Médico Legal de Santiago y H. Consejo de Monumentos Nacionales por gestionar la recuperación de los restos.

\section{REFERENCIAS BIBLIOGRÁFICAS}

AMBROSE, S. y M. KATZENBERG. 2000. Biogeochemical Approaches to Paleodietary Analysis. Advances in archaeological and museum science. Vol. 5. Kluwer Academic Publishers, New York.

BARBERENA, R. 2002. Los límites del mar. Isótopos estables en Patagonia meridional. Sociedad Argentina de Antropología, Buenos Aires.

BERÓN, M., E. BAFFI, R. MOLINARI, G. BARRIENTOS, C. ARANDA y L. LUNA. 2000. Estructuras funerarias de momentos tardíos en Pampa-Patagonia. El "chenque" de Lihué Calel. En: Desde el país de los gigantes. Perspectivas arqueológicas en Patagonia. Actas de las IV Jornadas de Arqueología de la Patagonia, pp: 141-159. UNPA, Río Gallegos.

BERÓN, M. y E. BAFFI. 2004. Variabilidad de las estructuras mortuorias en el Holoceno tardío. Cuenca de los lagos Posadas y Salitroso (prov. de Santa Cruz) y área de Lihué Calel (prov. de La Pampa). En: Contra Viento y Marea. Arqueología de Patagonia, pp: 387-402. Instituto Nacional de Antropología y Pensamiento Latinoamericano y Sociedad Argentina de Antropología, Buenos Aires.

BERÓN, M., L. LUNA y R. BARBERENA. 2009. Isotopic archaeology in the Western Pampas (Argentina): Preliminary results and perspectives. International Journal of Osteoarchaeology 19: 250-265.

BRONK RAMSEY, C. 2009. Bayesian analysis of radiocarbon dates. Radiocarbon 51(1): 337-360.

BROOKS, S. y J. SUCHEY. 1990. Skeletal age determination based on the os pubis: a comparision on the AcsádiNemeskéri and Suchey-Brooks Method. Human Evolution 5: 227-238.
BUIKSTRA, J. y D. UBELAKER. 1994. Standars for data collection fron human skeletal remains. Arkansas archaeological survey research series 44 .

CASSIODORO G. y S. GARCÍA. 2009. Análisis del registro tecnológico y osteológico de los entierros humanos del Holoceno tardío del lago Salitroso (Santa Cruz): un aporte al estudio del comportamiento mortuorio de cazadores-recolectores. En: Arqueología de la Patagonia, editado por M. Salemme, F. Santiago, M. Álvarez, E. Piana, M. Vásquez y E. Mansur, tomo 2: 613-628. Editorial Utopias, Ushuaia.

CASTRO, A. y J. MORENO. 2000. Noticia sobre enterratorios humanos en la costa norte de Santa Cruz - Patagonia Argentina. Anales del Instituto de la Patagonia. Serie Ciencias Humanas. Vol.28, pp 225- 231.

DE LA VAULX, H. 1901 Voyage en Patagonie. Hachette, Paris. GILL, G. y S. RHINE. 1990. Skeletal attribution of race. Methods for Forensic Antrophology. University of New Mexico, Albuquerque.

GOÑI, R. 2001 Fechados radiocarbónicos y registro arqueológico en la cuenca de los lagos Salitroso/Posadas (Santa Cruz). Cuadernos del INAPL 19: 666-668.

GOÑI, R. y G. BARRIENTOS. 2000 Estudio de chenques en Lago Salitroso, Provincia de Santa Cruz. En: Desde el país de los gigantes. Perspectivas arqueológicas en Patagonia. Actas de las IV Jornadas de Arqueología de la Patagonia, pp: 161-178. UNPA, Río Gallegos.

GOÑI, R. y G. BARRIENTOS. 2004. Poblamiento tardío y movilidad en la cuenca del lago Salitroso. En: Contra Viento y Marea. Arqueología de Patagonia, pp: 313324. Instituto Nacional de Antropología y Pensamiento Latinoamericano y Sociedad Argentina de Antropología, Buenos Aires.

GOÑI, R., G. BARRIENTOS, M. J. FIGUERERO, G. MENGONI, F. MENA, V. LUCERO y O. REYES. 2004. Distribución espacial de entierros en la cordillera de Patagonia centromeridional (Lago Salitroso-Paso Roballos, Argentina/ Entrada Baker-Chacabuco, Chile). En: Actas XV Congreso Nacional de Arqueología Chilena, tomo II:1101-1108, Chungara Revista de Antropología Chilena, Arica.

MORANO, S., V. SIERPE y A. PRIETO. 2009. Rescate del chenque de cerro Guido. Arqueología de la Patagonia, editado por M. Salemme, F. Santiago, M. Álvarez, E. Piana, M. Vásquez y E. Mansur, tomo 2: 661-668. Editorial Utopias, Ushuaia.

MEINDL, R. y C. O. LOVEJOY. 1985 Ectocranial suture closure: a revised method for the determination of skeletal age at death based on the lateral-anterior sutures. American journal of physical anthropology 68: 57-66. 
MENA, F. y V. LUCERO 2004. En torno a las últimas poblaciones indígenas de la cordillera centro-patagónica: estudio comparado de tres valles en Aisén oriental (Chile). En: Contra Viento y Marea. Arqueología de Patagonia, pp: 643-658. Instituto Nacional de Antropología y Pensamiento Latinoamericano y Sociedad Argentina de Antropología, Buenos Aires.

ORTNER, D. 2003. Identification of pathological conditions in human skeletal remains. Academic Press, San Diego.

PRIETO, A. 1993-94 Algunos datos en torno a los enterratorios humanos de la región continental de Magallanes. Anales del Instituto de la Patagonia. Serie Ciencias Humanas 22: 91-100.

REYES, O. 2001. Enterratorios indígenas en el curso inferior del valle del Río Ibáñez, Región de Aisén. Boletín de la Sociedad Chilena de Arqueología 31: 61-64.

-2002. Funebria indígena en el curso inferior del valle del río Ibáñez, margen occidental de la estepa centropatagónica (XI Región de Aisén). Anales del Instituto de la Patagonia. Serie Ciencias Humanas 30: 87-101. -2004. Contextos funerarios del Holoceno tardío en el margen oriental de la cordillera de Aisén (XI Región); curso inferior de los valles del río Ibáñez y Jeinemeni y curso superior del valle del río Chacabuco. Patagonia Central. Memoria de título de Arqueólogo. Departamento de Antropología, Universidad de Chile. MS.

REYES, O., C. MÉNDEZ, H. VELÁSQUEZ y V. TREJO. 2006.

Distribuciones espaciales y contextos arqueológicos de cazadores recolectores esteparios en alto río Cisnes (XI Región de Aisén). Magallania 34(2):75-90.

SERVICIO MÉDICO LEGAL. 2009. Informe Antropológico y Arqueológico. Protocolo № 95-08 U.E. Causa RUC № 0800871398-8. 04 de Junio de 2009, Santiago. MS.

TESSONE, A., F. ZANGRANDO, G. BARRIENTOS, S. VALENCIO, H. PANARELLO y R. GOÑI. 2005. Isótopos estables del carbono en Patagonia meridional: datos de la cuenca del lago Salitroso (Provincia de Santa Cruz, República Argentina). Magallania 33(2): 21-28.

TESSONE, A., F. ZANGRANDO, G. BARRIENTOS, R. GOÑI, H. PANARELLO y M. CAGNONI. 2009. Stable isotope studies in the Salitroso Lake Basin (Southern Patagonia, Argentina): assessing diet of late Holocene hunter-gatherers. International Journal of Osteoarchaeology 19: 297-308. 